\title{
Geospatial Correlation of
} Amyopathic Dermatomyositis With Fixed Sources of Airborne Pollution: A Retrospective Cohort Study

\author{
David R. Pearson ${ }^{1 *}$ and Victoria P. Werth ${ }^{2,3}$ \\ ${ }^{1}$ Department of Dermatology, University of Minnesota Medical School, Minneapolis, MN, United States, ${ }^{2}$ Corporal Michael J. \\ Crescenz VA Medical Center, Philadelphia, PA, United States, ${ }^{3}$ Department of Dermatology, Perelman School of Medicine, \\ University of Pennsylvania, Philadelphia, PA, United States
}

OPEN ACCESS

Edited by:

Karin Loser,

University of Münster, Germany

Reviewed by:

Takashi Hashimoto,

Osaka City University, Japan

Artem Vorobyev,

University Medical

Center Schleswig-Holstein, Germany

*Correspondence:

David R. Pearson

pearsond@umn.edu

Specialty section:

This article was submitted to

Dermatology,

a section of the journal

Frontiers in Medicine

Received: 06 February 2019

Accepted: 04 April 2019

Published: 24 April 2019

Citation:

Pearson DR and Werth VP (2019) Geospatial Correlation of Amyopathic Dermatomyositis With Fixed Sources of Airborne Pollution: A Retrospective Cohort Study. Front. Med. 6:85 doi: 10.3389/fmed.2019.00085
Objective: Dermatomyositis (DM) may result from exogenous triggers, including airborne pollutants, in genetically susceptible individuals. The United States Environmental Protection Agency's 2011 National Air Toxics Assessment (NATA) models health risks associated with airborne emissions, available by ZIP code tabulation area (ZCTA). Important contributors include point (fixed), on-road, and secondary sources. The objective of this study was to investigate the geospatial distributions of DM and subtypes, classic DM (CDM) and clinically amyopathic DM (CADM), and their associations with airborne pollutants.

Methods: This retrospective cohort study identified 642 adult DM patients from 336 unique ZCTAs. GeoDa v.1.10 was used to calculate global and local Moran's indices and generate local indicator of spatial autocorrelation (LISA) maps. All Moran's indices and LISA maps were permuted 999 times.

Results: Univariate global Moran's indices for DM, CDM, and CADM prevalence were not significant, but LISA maps demonstrated differential local spatial clustering and outliers. CADM prevalence correlated with point sources (bivariate global Moran's index 0.071 , pseudo- $p=0.018)$, in contrast to $\operatorname{CDM}(-0.0053$, pseudo- $p=0.46)$. Bivariate global Moran's indices for DM, CDM, and CADM prevalence did not correlate with other airborne toxics, but bivariate LISA maps revealed local spatial clustering and outliers.

Conclusion: Prevalence of CADM, but not CDM, is geospatially correlated with fixed sources of airborne emissions. This effect is small but significant and may support the hypothesis that triggering exposures influence disease phenotype. Important limitations are NATA data and ZCTA population estimates were collected from 2011 and ZCTA of residence may not have been where patients had greatest airborne pollutant exposure.

Keywords: dermatomyositis, pollution, environmental, geospatial analysis, Moran index

\section{INTRODUCTION}

The pathogenesis of dermatomyositis (DM) is incompletely understood, but may result from diverse exogenous exposures that trigger disease in genetically susceptible individuals, including drugs, infections, and environmental factors such as ultraviolent radiation (UVR) and airborne pollutants (1-18). Important subtypes include classic DM (CDM), which demonstrates clinical and 
laboratory evidence of myositis, and clinically amyopathic DM (CADM), diagnosed when patients do not have symptomatic myositis and have only minimal or no objective findings of myositis $(19,20)$. There is evidence that disease phenotype has been associated with specific exposure patterns $(5,8,10,21)$.

The United States Environmental Protection Agency (EPA), an independent agency of the United States federal government that aims to protect human health and the environment, provides publicly available data on airborne emissions in the United States at the level of ZIP code tabulation areas (ZCTA) (22). ZCTAs are geographic representations of the United States Postal Service ZIP code service areas, and were developed by the United States Census Bureau to tabulate summary statistics over geographic areas. In 2015, the EPA released the most recent version of the National Air Toxics Assessment (NATA) based on 2011 emissions. NATA is a screening tool designed to provide information on potential risks of breathing air toxics (22). Emission levels of 181 air toxics (such as benzene, formaldehyde, and diesel particulate matter) were measured, then modeled to predict overall health risks and applied over geographic areas (22). Important contributors include point sources, which denote emissions from larger, stationary industrial and commercial facilities, on-road sources, produced by vehicles on roads, and secondary sources, which are formed from the chemical reactions of other emitted pollutants (22). Since DM has been associated with airborne pollutants, this dataset may be useful in analyzing exposure patterns (11-13).

Geospatial analysis is a statistical approach to analyze data over a geographic region, with applications in environmental science, public health, and other fields. Geospatial statistical techniques, including global and local Moran's indices, can be used to evaluate clustering and dispersion patterns, and have found increasing use in medicine (23-25). These techniques have not been applied to the study of DM due in part to the low incidence and prevalence of disease. The relatively large cohort at the University of Pennsylvania provides an opportunity to use geospatial analysis to assess exposure patterns in DM.

The objective of this study was to investigate the geospatial distributions of DM and its subtypes and their associations with airborne pollutants in the greater Philadelphia metropolitan area.

\section{METHODS}

\section{Patient Selection}

This retrospective cohort study spanned January 1, 2000 through December 31, 2017. Data collection was complete July 31, 2018. Adult patients $\geq 18$ years of age seen in dermatology or rheumatology clinics at the University of Pennsylvania with a United States ZCTA of primary residence listed in their medical record and a diagnosis of DM, encoded by International Classification of Disease (ICD) 9th and 10th revision codes (710.3 and M33.0X, M33.1X, M33.9X, respectively) were included. Patients were identified using PennSeek, a custom, secure implementation of Oracle's web-based Endeca Information Discovery platform adapted by the University of Pennsylvania Data Analytics Core. PennSeek allows for targeted keyword searches using Boolean logic of unstructured or semi-structured medical documents that reside in the main Penn Medicine electronic health record and ancillary systems, described in detail elsewhere (26). After assembling the cohort, individual patient charts were reviewed to verify diagnosis, exclude erroneously coded patients, and extract variables of interest.

\section{NATA Dataset}

2011 NATA risk estimates including total airborne, point, onroad, and secondary source risks were taken from the EPA's dataset, organized by ZCTA (22).

\section{Variables}

DM subtype was assigned based on the treating clinician's note. In cases of ambiguity or disagreement, evidence of myositis was determined by patient-reported symptoms plus an objective sign (elevated creatinine kinase or aldolase or consistent EMG, MRI, or muscle biopsy). Patients who fulfilled these criteria were labeled as CDM and those lacking labeled as CADM. Demographics and ZCTAs were extracted from the medical record. Prevalence was calculated using United States Census Bureau (USCB) 2007-2011 American Community Survey population estimates, organized by ZCTA (27).

\section{Analysis}

Continuous variables are presented as median (interquartile range, IQR) and categorical data are presented as count (percentage). Heatmaps were created with Tableau Public version 10.4 (Tableau Software, Inc. 2018. Seattle, Washington).

Geospatial analysis was performed by calculation of the global Moran's index, an inferential statistic which estimates spatial autocorrelation and falls between -1 and +1 (28). More positive or negative values correspond to greater spatial clustering or competitive dispersion, respectively (28). To test the null hypothesis of random spatial distribution, empiric significance is computed by permutation to yield a pseudo$p$-value (28). A pseudo- $p$-value differs from an analytic $p$ value because it is a summary of the results of the reference distribution, and is dependent on the number of permutations used (28). In order to visualize spatial clustering, local indicators of spatial autocorrelation (LISA) maps were created using local Moran's index calculations (29). This statistic identifies significant locations as high-high or low-low spatial clusters and high-low or low-high spatial outliers relative to neighboring regions (29). In both indices, neighbor less regions are excluded from analysis.

Bivariate global and local Moran's indices were calculated in the same fashion; global indices measure spatial autocorrelation between an outcome variable in one geographic region and a second variable in neighboring regions, and local indices were mapped with bivariate LISA (BiLISA) maps (30).

GeoDa version 1.10 was used for geospatial analyses. Shapefiles were obtained from the USCB 2017 ZCTA boundary files and the weights matrix was assigned first-order queen contiguity (neighboring regions share common edges or vertices). All global and local univariate and bivariate Moran's indices and LISA and BiLISA maps were permuted 999 times. A pseudo- $p<0.05$ was set as significant. 


\section{Ethics}

This study was carried out in accordance with the recommendations of the University of Pennsylvania Institutional Review Board (protocol number 828959), which waived the requirement of written informed consent from subjects. Written consent was waived due to the retrospective nature of the study and minimal risk it posed to subjects. The protocol for this study conforms to the ethical guidelines of the Declaration of Helsinki. The protocol was approved by the University of Pennsylvania Institutional Review Board.

\section{RESULTS}

Of 205,084 adult patients seen in dermatology or rheumatology clinics at the University of Pennsylvania from January 1, 2000 through December 31, 2017, PennSeek identified 653 patients. Upon individual chart review, 9 patients were excluded for diagnoses other than DM and 2 were excluded for absence of a ZCTA in their medical record, resulting in 642 patients who met inclusion criteria.

Baseline characteristics of the patient cohort are noted in Table 1. The median age at symptom onset was 49 (IQR 37, 58) years. Most patients were female $(532,82.8 \%)$, white $(476,74.1 \%)$, and diagnosed with $\operatorname{CDM}(439,68.4 \%)$, though a notable fraction had CADM $(203,31.6 \%)$. Data on year of symptom onset was available for 630 patients (98.1\%) and divided into approximate quartiles. One hundred fifty-nine patients (25.2\%) developed symptoms in 2005 or earlier, 176 patients (27.9\%) between 2006 and 2009, 153 patients between 2010 and 2013, and 142 patients (22.5\%) from 2014 to the end of the study period.

Three hundred thirty-six unique ZCTAs represented a total underlying population of 8,110,198 (Table 1, Data Sheet 1). The median prevalence of DM per ZCTA was 8.6 (IQR 4.6, 15.2) per 100,000 . When ZCTAs outside of the greater Philadelphia metropolitan area were excluded, the median prevalence of DM was 9.2 (IQR 5.2, 16.3) per 100,000. The median total airborne risk per ZCTA was 43 (IQR 37, 48) per million, including 1.24 (IQR 0.64, 1.67) per million from point sources, 9.13 (IQR 6.56, 12.18) per million from on-road sources, and 18.10 (IQR 16.47, 19.17) per million from secondary sources.

Heatmaps of the prevalence of DM and subtypes CDM and CADM in the greater Philadelphia metropolitan area are illustrated in Figure 1, divided into sextiles. Many ZCTAs with high prevalence of DM and CDM were observed in the western and northern metropolitan area. CADM prevalence is lower overall and aligns along a northeast-southwest axis. Figure $\mathbf{S 1}$ shows heatmaps of the onset year by quartile, with no clear patterns of clustering or outliers.

\section{Geospatial Analysis}

Univariate global Moran's indices for the prevalence of DM, CDM, and CADM were not significant (Table 2), but LISA maps demonstrated differential local spatial clustering and outliers (Figure 2), as indicated by the presence of high-high (red) and low-low clustering (bright blue), and high-low (pink) and lowhigh (light blue) outliers. DM and CDM shared several clustered
TABLE 1 | Baseline characteristics of adult dermatology and rheumatology patients with dermatomyositis (DM) seen at the University of Pennsylvania between January 1, 2000 and December 31, 2017, with median 2011 NATA risk calculations.

\begin{tabular}{|c|c|}
\hline Metric & Cohort $(n=642)$ \\
\hline Median age at symptom onset, years (IQR) & $49(37,58)$ \\
\hline Female sex & $532(82.8 \%)$ \\
\hline \multicolumn{2}{|l|}{ PATIENT-REPORTED RACE/ETHNICITY } \\
\hline White & $476(74.1 \%)$ \\
\hline Black & $76(11.8 \%)$ \\
\hline Asian, American Indian, or Alaskan Native & $20(3.1 \%)$ \\
\hline Other & 70 (10.9\%) \\
\hline \multicolumn{2}{|l|}{ DM SUBTYPE } \\
\hline CDM & $439(68.4 \%)$ \\
\hline CADM & $203(31.6 \%)$ \\
\hline \multicolumn{2}{|c|}{ SYMPTOM ONSET YEAR QUARTILES $(n=630)$} \\
\hline 2005 or earlier & 159 (25.2\%) \\
\hline 2006-2009 & $176(27.9 \%)$ \\
\hline 2010-2013 & $153(24.3 \%)$ \\
\hline 2014-2017 & $142(22.5 \%)$ \\
\hline \multicolumn{2}{|l|}{ ZCTA } \\
\hline Unique ZCTAs & 336 \\
\hline 2010 Census population & $8,110,198$ \\
\hline $\begin{array}{l}\text { Median DM prevalence per ZCTA (IQR), per } \\
100,000\end{array}$ & $8.6(4.6,15.2)$ \\
\hline $\begin{array}{l}\text { Median DM prevalence per Phl metropolitan } \\
\text { ZCTA (IQR), per 100,000 }(n=607)\end{array}$ & $9.2(5.2,16.3)$ \\
\hline \multicolumn{2}{|c|}{ MEDIAN 2011 NATA RISK PER ZCTA, PER MILLION } \\
\hline Total airborne risk (IQR) & $43(37,48)$ \\
\hline Point source risk (IQR) & $1.24(0.64,1.67)$ \\
\hline On-road source risk (IQR) & $9.13(6.56,12.18)$ \\
\hline Secondary source risk (IQR) & $18.10(16.47,19.17)$ \\
\hline
\end{tabular}

CADM, clinically amyopathic dermatomyositis; CDM, classic dermatomyositis; DM, dermatomyositis; IQR, interquartile range; NATA, National Air Toxics Assessment; Phl, Philadelphia; ZCTA, zip code tabulation area.

and outlier ZCTAs, and differed notably from CADM, which demonstrated high-high clustering along a northeast-southwest axis. High-low outliers occurred predominantly in ZCTAs of low relative population.

The prevalence of CADM vs. point sources showed a significant bivariate global Moran's index (0.071, pseudo$p=0.02$ ), and BiLISA mapping demonstrating western metropolitan high-high clustering and eastern metropolitan lowlow clustering (Figure 3a). In contrast, the prevalence of CDM vs. point sources was not significant $(-0.0053$, pseudo- $p=0.46)$, and BiLISA mapping demonstrating western low-high outliers and eastern low-low clustering (Figure 3b). Bivariate global Moran's indices for the prevalence of DM, CDM, and CADM vs. other airborne toxics were not significant, and BiLISA mapping revealed similar local spatial clustering and outliers between subtypes (Figure S2).

Univariate global Moran's indices for both DM onset year (by quartile) and median age at symptom onset were not significant (data not shown). 

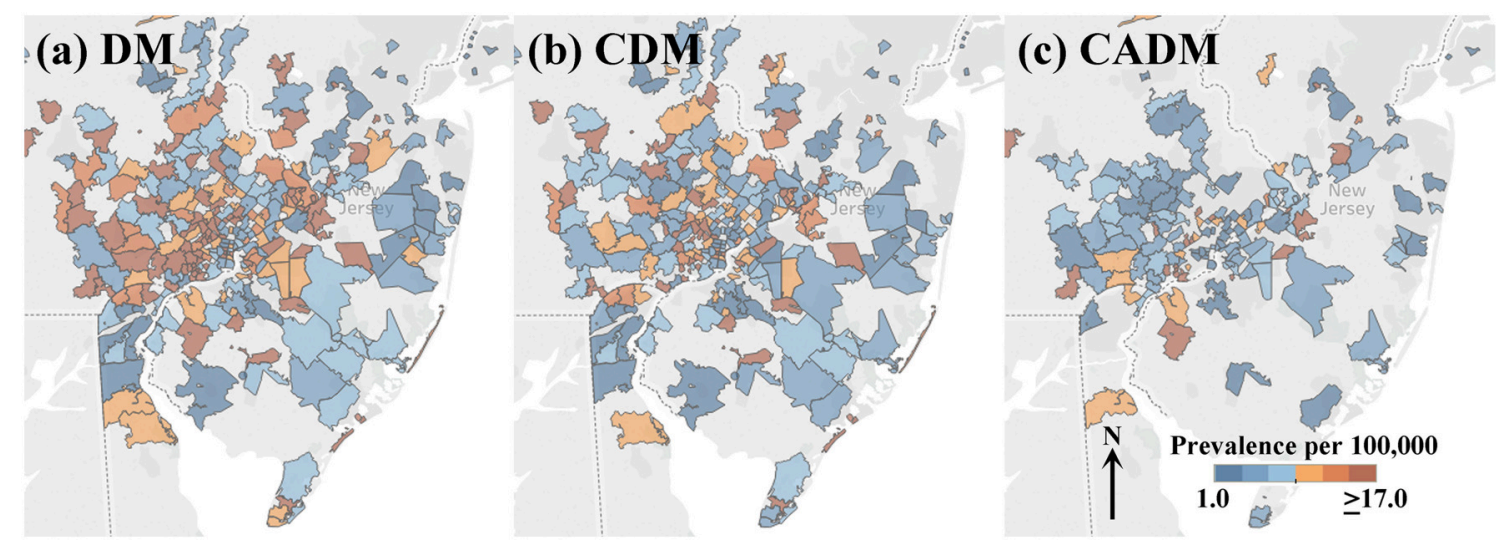

FIGURE 1 | Heatmaps of prevalence per 100,000 of the full cohort of dermatomyositis (DM) patients (a), and subtypes classic DM (CDM, b) and clinically amyopathic DM (CADM, c) in the greater Philadelphia metropolitan area (legend inset: low prevalence, blue; high prevalence, orange). Many ZCTAs with high prevalence of DM and CDM are observed in the western and northern metropolitan area. CADM prevalence is lower across the region and aligns along a northeast-southwest axis.

TABLE 2 | Univariate global Moran's indices of the prevalence of dermatomyositis (DM) and subtypes, and bivariate global Moran's indices of DM prevalence vs. 2011 NATA metrics.

\begin{tabular}{llcc}
\hline $\begin{array}{l}\text { Outcome } \\
\text { variable }\end{array}$ & $\begin{array}{l}\text { Lagged } \\
\text { variable }\end{array}$ & $\begin{array}{c}\text { Global Moran's } \\
\text { index }\end{array}$ & Pseudo $\boldsymbol{p}$-value \\
\hline DM prevalence & Univariate & 0.0054 & 0.16 \\
& Total airborne risk & 0.0080 & 0.34 \\
& Point sources $^{*}$ & 0.0019 & 0.36 \\
& On-road sources* $^{*}$ & 0.0057 & 0.15 \\
& Secondary sources* & 0.0036 & 0.46 \\
CDM prevalence & Univariate & 0.0051 & 0.16 \\
& Total airborne risk* & 0.0080 & 0.33 \\
& Point sources* & -0.0053 & 0.46 \\
& On-road sources* & 0.0082 & 0.28 \\
& Secondary sources* & -0.00076 & 0.47 \\
CADM prevalence & Univariate & 0.064 & 0.06 \\
& Total airborne risk & -0.0015 & 0.49 \\
& Point sources & $\mathbf{0 . 0 7 1}$ & $\mathbf{0 . 0 2}$ \\
& On-road sources & & 0.18 \\
& Secondary sources* & -0.025 & 0.09 \\
\hline
\end{tabular}

CADM, clinically amyopathic dermatomyositis; CDM, classic dermatomyositis; $D M$, dermatomyositis; NATA, National Air Toxics Assessment. *Bivariate global Moran's index.

Bold values indicate significant finding.

\section{DISCUSSION}

In this retrospective study of $642 \mathrm{DM}$ patients, we found a significant geospatial correlation between exposure to point sources of airborne pollutants and CADM. Other airborne toxics including overall calculated airborne risk, on-road, and secondary sources did not correlate with DM, CDM, or CADM prevalence.

The association between point sources and CADM is modest but significant, as seen by the magnitude of the global Moran's index. Since DM has been associated with a variety of exogenous triggers besides airborne toxics, it is not surprising that this exposure does not account for all cases. It is noteworthy that CDM was not correlated with point sources, supporting the hypothesis that the type of triggering exposure may influence disease phenotype.

Past studies have demonstrated associations between specific exogenous triggers and serotype, which is known to correlate with clinical phenotype (31-33). The intensity of UVR exposure has been strongly associated with the proportion of CDM patients expressing anti-Mi-2 antibodies (odds ratio [OR] 6.0), while antiTIF1- $\gamma$ antibodies have been negatively correlated with latitude (OR 0.96) $(8,21)$. HLA alleles associated with anti-Mi-2 and antiTIF1- $\gamma$ antibodies were also negatively associated with latitude (21). Anti-MDA-5 antibodies were inversely associated with population of city of residence and appeared to cluster along rural areas near the Kiso River in Japan (10). Hydroxyurea may induce a DM-like eruption that spares muscles, while other drugs may induce a CDM-like presentation and viral infections may trigger juvenile DM $(5,34)$.

This study has important limitations. It is a retrospective cohort from a tertiary referral facility in a heavily-populated urban center. The ZCTA of residence listed in the patient's chart may not have been the location where greatest airborne pollutant exposure occurred and does not factor transportation routes or job location. The listed ZCTA may not have been where DM onset occurred, especially in longstanding cases. Reference data were taken from available years: ZCTAs were collected from medical records in 2018, ZCTA shapefiles were based on 2017 USCB boundary files, NATA metrics were calculated from data collected in 2011, and ZCTA population estimates were collected from 2007 to 2011 in the USCB American Community Survey. Both population estimates and airborne pollutant levels may have changed since 2011, though more recent, reliable data was not available.

It is important to note that NATA metrics are risk assessments based on collected airborne emissions and do not reflect exposure to all compounds, all pathways of exposure, or accurately model 


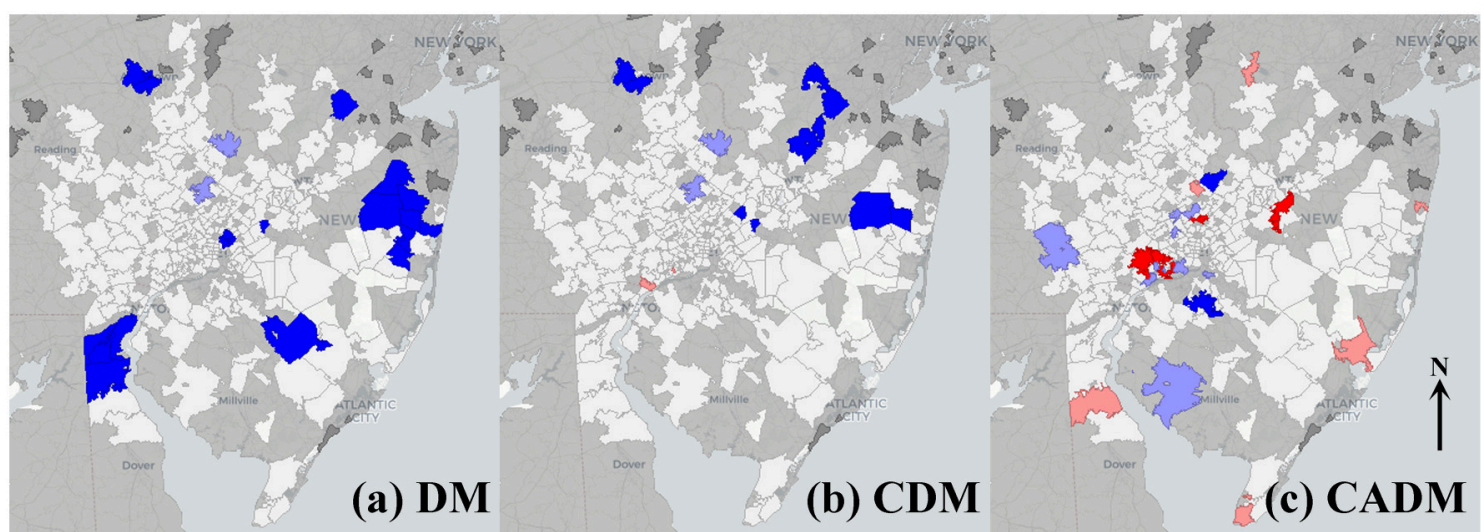

FIGURE 2 | Local indicators of spatial autocorrelation (LISA) maps for prevalence of the full cohort of dermatomyositis (DM) patients (a), and subtypes classic DM (CDM, b) and clinically amyopathic DM (CADM, c). High-high (red) and low-low (bright blue) spatial clusters, and high-low (pink) and low-high (light blue) spatial outliers relative to neighboring regions are identified. Geospatially non-significant (white) and neighborless (dark gray) regions are visible. Differential spatial clustering is most notable between CDM (b) and CADM (c).

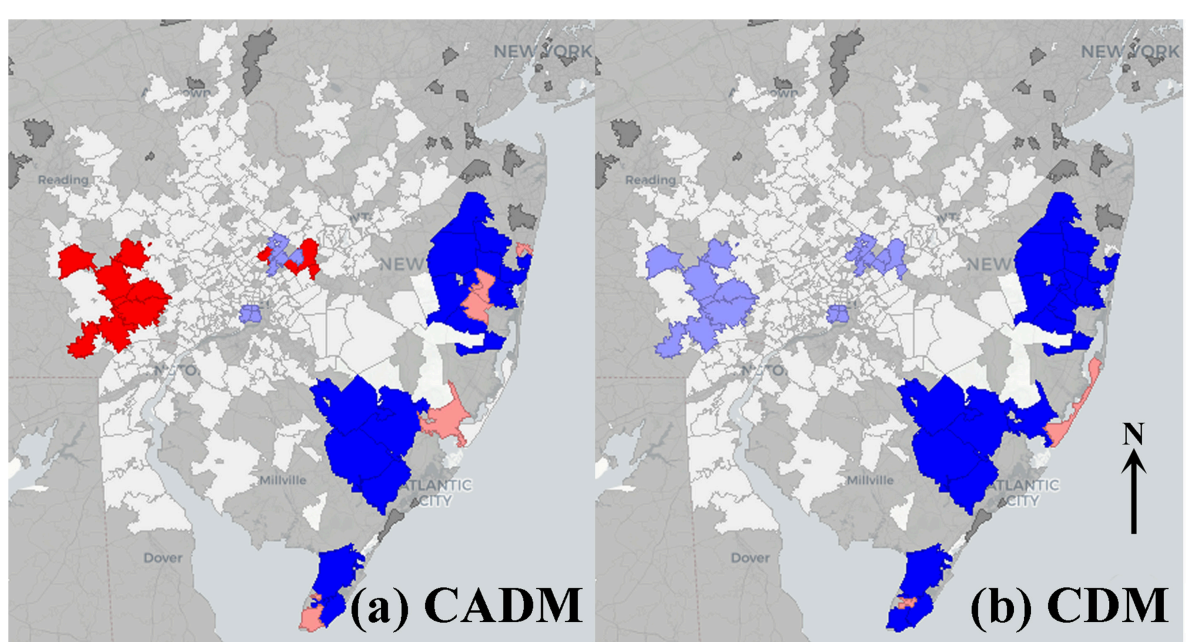

FIGURE 3 | Bivariate local indicators of spatial autocorrelation (BiLISA) maps for prevalence of clinically amyopathic dermatomyositis (CADM, a) and classic dermatomyositis (CDM, b) vs. point sources. High-high (red) and low-low (bright blue) spatial clusters, and high-low (pink) and low-high (light blue) spatial outliers relative to neighboring regions are identified. In CADM (a), western metropolitan high-high clustering and eastern metropolitan low-low clustering is prominent, while in CDM (b), western low-high outliers and eastern low-low clustering is notable. Geospatially non-significant (white) and neighbor less (dark gray) regions are visible.

episodic emissions (22). Risk assessments by source represent pooled data from many pollutants, and the contributed risks of individual compounds were not assessed. Furthermore, these data apply to geographic areas and groups, rather than specific locations or individuals (22).

The prevalence of DM found in this study is lower than previously reported (35). This estimate is not population-based and likely underestimates the true prevalence in the greater Philadelphia metropolitan area. During the 18-year time period of this study, there were 5 major academic medical centers and a large number of group and individual dermatology and rheumatology practices, many of whom see DM patients not included here.

While the spatial distribution of onset of DM symptoms changed over time, specific regions did not cluster by onset year.
DM frequency remained approximately stable when comparing the 4-year intervals from 2006 to 2009, 2010 to 2013, and 2014 to 2017. However, DM, especially CADM, is frequently delayed in diagnosis or misdiagnosed and presentation to a tertiary referral center may result in additional delay, so patients with more recent onset may be unaccounted for (36).

Identifying exogenous triggers for DM is challenging given the heterogeneity of both exposure and the underlying disease. Geospatial analysis is a candidate method for studying geographic patterns of exposure and may have applications examining infectious triggers, pollutants, demographics, and other factors. Future population-based studies may better estimate these associations. Further exploration of the geospatial distribution of DM phenotype by serology, malignancy association, or the presence of interstitial lung disease may 
also be useful for determining risk factors and understanding disease pathogenesis.

In conclusion, in this retrospective cohort study we found prevalence of CADM, but not CDM, is geospatially correlated with larger, geographically fixed sources of airborne pollution. This effect is small but significant and may support the hypothesis that triggering exposures may influence disease phenotype in DM.

\section{ETHICS STATEMENT}

This study was carried out in accordance with the recommendations of the University of Pennsylvania Institutional Review Board (protocol number 828959), which waived the requirement of written informed consent from subjects. Written consent was waived due to the retrospective nature of the study and minimal risk it posed to subjects. The protocol for this study conforms to the ethical guidelines of the Declaration of Helsinki. The protocol was approved by the University of Pennsylvania Institutional Review Board.

\section{AUTHOR CONTRIBUTIONS}

DP and VW contributed conception and design of the study. DP collected the primary data, performed the statistical analysis, and wrote the first draft of the manuscript. DP and VW contributed to manuscript revision and read and approved the submitted version.

\section{REFERENCES}

1. Hengstman GJD, Van Venrooij WJ, Vencovsky J, Moutsopoulos HM, Van Engelen BGM. The relative prevalence of dermatomyositis and polymyositis in Europe exhibits a latitudinal gradient. Ann Rheum Dis. (2000)59:141-2. doi: 10.1136/ard.59.2.141

2. Neil Crowson A, Magro CM, Dawood MR. A causal role for parvovirus B19 infection in adult dermatomyositis and other autoimmune syndromes. J Cutan Pathol. (2000)27:505-15. doi: 10.1034/j.1600-0560.2000.027010505.x

3. Okada S, Weatherhead E, Targoff IN, Wesley R, Miller FW. Global surface ultraviolet radiation intensity may modulate the clinical and immunologic expression of autoimmune muscle disease. Arthritis Rheum. (2003)48:228593. doi: 10.1002/art.11090

4. Packman LM, Lipton R, Ramsey-Goldman R, Shamiyeh E, Abbott K, Mendez EP, et al. History of infection before the onset of juvenile dermatomyositis: results from the National Institute of Arthritis and Musculoskeletal and Skin diseases research registry. Arthritis Care Res. (2005)53:166-72. doi: 10.1002/art.21068

5. Seidler AM, Gottlieb AB. Dermatomyositis induced by drug therapy: a review of case reports. J Am Acad Dermatol. (2008)59:872-80. doi: 10.1016/j.jaad.2008.05.034

6. Dastmalchi M, Grundtman C, Alexanderson H, Mavragani CP, Einarsdottir H, Helmers SB, et al. A high incidence of disease flares in an open pilot study of infliximab in patients with refractory inflammatory myopathies. Ann Rheum Dis. (2008)67:1670-7. doi: 10.1136/ard.2007.077974

7. Orbach H, Tanay A. Vaccines as a trigger for myopathies. Lupus. (2009)18:1213-6. doi: 10.1177/0961203309345734

8. Love LA, Weinberg CR, McConnaughey DR, Oddis CV, Medsger TA, Jr., Reveille JD, et al. Ultraviolet radiation intensity predicts the relative

\section{FUNDING}

This work was supported by the United States Department of Veterans Affairs (Veterans Health Administration, Office of Research and Development and Biomedical Laboratory Research and Development).

\section{SUPPLEMENTARY MATERIAL}

The Supplementary Material for this article can be found online at: https://www.frontiersin.org/articles/10.3389/fmed. 2019.00085/full\#supplementary-material

Data Sheet 1 | Unique ZCTAs and corresponding census populations and DM counts, calculated prevalences, and NATA risk estimates are shown. On the far right, counts of DM are listed by year of symptom onset. CADM, clinically amyopathic dermatomyositis; CDM, classic dermatomyositis; DM, dermatomyositis; NATA, National Air Toxics Assessment; ZCTA, zip code tabulation area.

Figure S1 | Heatmaps of new-onset cases of dermatomyositis in the greater Philadelphia metropolitan area in 2005 or earlier (a), 2006-2009 (b), 2010-2013 (c), and 2014-2017 (d) (legend inset: lower count, light blue; higher count, dark blue). Geospatial analysis did not demonstrate clustering or dispersion.

Figure S2 | Bivariate local indicators of spatial autocorrelation (BiLISA) maps for prevalence of the full cohort of dermatomyositis (DM) and subtypes classic DM (CDM) and clinically amyopathic DM (CADM) vs. total airborne risk (a-c, respectively), on-road sources (d-f, respectively), and secondary sources (g-I, respectively). High-high (red) and low-low (bright blue) spatial clusters, and high-low (pink) and low-high (light blue) spatial outliers relative to neighboring regions are identified. Geospatially non-significant (white) and neighbor less (dark gray) regions are visible. Note the similarities in both spatial clustering and outliers between DM, CDM, and CADM. All global Moran's indices were non-significant.

distribution of dermatomyositis and anti-Mi-2 autoantibodies in women. Arthritis Rheum. (2009) 60:2499-504. doi: 10.1002/art.24702

9. Rider LG, Wu L, Mamyrova G, Targoff IN, Miller FW. Environmental factors preceding illness onset differ in phenotypes of the juvenile idiopathic inflammatory myopathies. Rheumatology. (2010) 49:2381-90. doi: 10.1093/rheumatology/keq277

10. Muro Y, Sugiura K, Hoshino K, Akiyama M, Tamakoshi K. Epidemiologic study of clinically amyopathic dermatomyositis and anti-melanoma differentiation-associated gene 5 antibodies in central Japan. Arthritis Res Ther. (2011) 13:6. doi: 10.1186/ar3547

11. Orione MA, Silva CA, Sallum AM, Campos LM, Omori CH, Braga $\mathrm{AL}$, et al. Risk factors for juvenile dermatomyositis: exposure to tobacco and air pollutants during pregnancy. Arthritis Care Res. (2014)66:1571-5. doi: 10.1002/acr.22358

12. Blanc $\mathrm{PD}$, Järvholm $\mathrm{B}$, Torén $\mathrm{K}$. Prospective risk of rheumatologic disease associated with occupational exposure in a cohort of male construction workers. Am J Med. (2015)128:1094-101. doi: 10.1016/j.amjmed.2015.05.001

13. Bernatsky S, Smargiassi A, Barnabe C, Svenson LW, Brand A, Martin $\mathrm{RV}$, et al. Fine particulate air pollution and systemic autoimmune rheumatic disease in two Canadian provinces. Environ Res. (2016)146:85-91. doi: 10.1016/j.envres.2015.12.021

14. Wright NA, Vleugels RA, Callen JP. Cutaneous dermatomyositis in the era of biologicals. Semin Immunopathol. (2016)38:113-21. doi: 10.1007/s00281-015-0543-z

15. Limaye V, Smith C, Koszyca B, Blumbergs P, Otto S. Infections and vaccinations as possible triggers of inflammatory myopathies. Muscle Nerve. (2017)56:987-9. doi: 10.1002/mus.25628

16. Mamyrova G, Rider LG, Ehrlich A, Jones O, Pachman LM, Nickeson $\mathrm{R}$, et al. Environmental factors associated with disease flare in 
juvenile and adult dermatomyositis. Rheumatology. (2017)56:1342-7. doi: 10.1093/rheumatology/kex162

17. Thompson C, Piguet V, Choy E. The pathogenesis of dermatomyositis. $\mathrm{Br} \mathrm{J}$ Dermatol. (2017) 179:1256-62. doi: 10.1111/bjd.15607

18. Zeidi M, Chansky P, Werth V. Acute onset/exacerbation of dermatomyositis and Sjogrens Syndrome following ingestion of IsaLean ${ }^{\circledR}$ herbal supplement: clinical and immunostimulatory findings. J Invest Dermatol. (2018)138:S75. doi: 10.1016/j.jid.2018.03.449

19. Bohan A, Peter J. Bohan A, Peter J. Polymyositis and dermatomyositis. N Engl J Med. (1975)292:344-7. doi: 10.1056/NEJM197502202920807

20. Sontheimer RD. Dermatomyositis: an overview of recent progress with emphasis on dermatologic aspects. Dermatol Clin. (2002)20:387-408. doi: 10.1016/S0733-8635(02)00021-9

21. Parkes J, Rothwell S, Oldroyd A, Chinoy H, Lamb J, (MYOGEN) MGC. Genetic background may contribute to the latitude-dependent prevalence of dermatomyositis and anti-TIF1- $\gamma$ autoantibodies in adult patients with myositis. Arthritis Res Ther. (2018)20:117. doi: 10.1186/s13075-018$1617-9$

22. United States Environmental Protection Agency. National Air Toxics Assessment. Available online at: https://www.epa.gov/nationalair-toxics-assessment (accessed March 26, 2019).

23. Toledo CRS de, Almeida AS de, Chaves SA de M, Sabroza PC, Toledo LM, Caldas JP. Vulnerability to the transmission of human visceral leishmaniasis in a Brazilian urban area. Rev Saude Publica. (2017)51:49. doi: 10.1590/s1518-8787.2017051006532

24. Dantas AP, De Azevedo UN, Nunes AD, Amador AE, Marques M V., Barbosa IR. Analysis of suicide mortality in brazil: spatial distribution and socioeconomic context. Rev Bras Psiquiatr. (2018)40:12-8. doi: 10.1590/1516-4446-2017-2241

25. Bagheri N, Wangdi K, Cherbuin N, Anstey KJ. General practice clinical data help identify dementia hotspots: a novel geospatial analysis approach. $J$ Alzheimers Dis. (2017)61:1. doi: 10.3233/JAD-170079

26. Small AM, Kiss DH, Zlatsin Y, Birtwell DL, Williams H, Guerraty MA, et al. Text mining applied to electronic cardiovascular procedure reports to identify patients with trileaflet aortic stenosis and coronary artery disease. J Biomed Inform. (2017)72:77-84. doi: 10.1016/j.jbi.2017.06.016

27. United States Census Bureau. American Fact Finder: American Community Survey. Available online at: https://factfinder.census.gov/faces/nav/jsf/pages/ programs.xhtml?program=acs. Published 2018.
28. Anselin L. Global Spatial Autocorrelation (1): Moran Scatter Plot and Spatial Correlogram. Available online at: https://geodacenter.github.io/workbook/5a global_auto/lab5a.html\#fn3 (accessed March 26, 2019).

29. Anselin L. Local Indicators of Spatial Association-LISA. Geogr Anal. (1995)27:93-115. doi: 10.1111/j.1538-4632.1995.tb00338.x

30. Anselin L. Global Spatial Autocorrelation : Bivariate, Differential and EB Rate Moran Scatter Plot. Available online at: https:/geodacenter.github.io/ workbook/5b_global_adv/lab5b.html (accessed March 26, 2019).

31. Daly ML, Gordon PA, Creamer D. Cutaneous features of dermatomyositis associated with myositis-specific antibodies. Br J Dermatol. (2017)176:1662-5. doi: 10.1111/bjd.15020

32. Best M, Jachiet M, Molinari N, Manna F, Girard C, Pallure V, et al. Distinctive cutaneous and systemic features associated with specific antimyositis antibodies in adults with dermatomyositis: a prospective multicentric study of 117 patients. J Eur Acad DermatolVenereol. (2018) 32:1164-72. doi: $10.1111 / j \mathrm{jv} .14759$

33. Wolstencroft PW, Fiorentino DF. Dermatomyositis clinical and pathological phenotypes associated with myositis-specific autoantibodies. Curr Rheumatol Rep. (2018) 20:5. doi: 10.1007/s11926-018-0733-5

34. Manlhiot C, Liang L, Tran D, Bitnun A, Tyrrell PN, Feldman BM. Assessment of an infectious disease history preceding juvenile dermatomyositis symptom onset. Rheumatology. (2008) 47:526-9. doi: 10.1093/rheumatology/ken038

35. Bendewald MJ, Wetter DA, Li X, Davis MDP. Incidence of dermatomyositis and clinically amyopathic dermatomyositis: a population-based study in Olmsted County, Minnesota. Arch Dermatol. (2010) 146:26-30. doi: 10.1001/archdermatol.2009.328

36. Da Silva D, Patel B, Werth V. Dermatomyositis: a diagnostic dilemma. J Am Acad Dermatol. (2018) 79:371-3. doi: 10.1016/j.jaad.2017.12.074

Conflict of Interest Statement: The authors declare that the research was conducted in the absence of any commercial or financial relationships that could be construed as a potential conflict of interest.

Copyright (๑) 2019 Pearson and Werth. This is an open-access article distributed under the terms of the Creative Commons Attribution License (CC BY). The use, distribution or reproduction in other forums is permitted, provided the original author(s) and the copyright owner(s) are credited and that the original publication in this journal is cited, in accordance with accepted academic practice. No use, distribution or reproduction is permitted which does not comply with these terms. 